Jurnal Kejuruteraan SI 3(1) 2020: 7-14

https://doi.org/10.17576/jkukm-2020-si3(1)-02

\title{
X-ray Sources Population in NGC 1559
}

\author{
Nurnabilah Nazria \& Adlyka Annuar ${ }^{a *}$ \\ ${ }^{a}$ Department of Applied Physics, Faculty of Science \& Technology, Universiti Kebangsaan Malaysia, \\ 43600 Bangi, Selangor, Malaysia. \\ *Corresponding author: adlyka@ukm.edu.my
}

Received 29 January 2020, Received in revised form 10 April 2020

Accepted 15 May 2020, Available online 30 September 2020

\begin{abstract}
We present Chandra observation of the nearby spiral galaxy NGC 1559 to study the X-ray sources population in the galaxy. Based on our analysis, we detect twenty-four X-ray sources in the galaxy, of which six have 0.5-8 keV luminosities exceeding $10^{39} \mathrm{erg} \mathrm{s}^{-1}$, suggesting that they are possibly ultra-luminous X-ray source (ULX) candidates. The remaining eighteen sources have $0.5-8 \mathrm{keV}$ luminosities below this threshold value, suggesting that they are likely to be X-ray binary candidates. We perform X-ray spectral analysis for the six ULX candidates and found that in general, their properties are broadly consistent with that found for other ULXS. The number of X-ray sources found in this galaxy is relatively high as compared to other galaxies with similar star formation rate of $\sim 2.8 M_{-} y r^{1}$. This however could be attributed to the relatively high number of supernova occurrence (four) in the galaxy observed in the past years. We did not detect any X-ray nuclear point source in the galaxy, suggesting that NGC 1559 does not host an active galactic nucleus. This is supported by its HII optical spectral classification and our infrared analysis using WISE.
\end{abstract}

Keywords: X-ray sources; NGC 1559; Chandra

\section{INTRODUCTION}

NGC 1559 is one of the closest galaxies to our own, located at a distance of $12.7 \mathrm{Mpc}$ with an optical spectral type of HII region (Goulding \& Alexander 2009). It is a late-type galaxy (Scd), with a relatively high star formation rate in the massive spiral arm, and strong radio flux emitting from the bar and the disc of the galaxy (Beck et al. 2002). Four supernova explosions have been detected in the galaxy; i.e., SNe 1984J (Evans et al. 1984), SNe 1986L (Mack et al. 1986), SN 2005df (Wang \& Baade 2005) and SN 2009ib (Pignata et al. 2009). All of them are classified as Type-II supernovae, which can result in the formation of binary systems consisting of compact stellar remnants such as white dwarf, neutron star and black hole. These can correlate to a relatively high number of off-nuclear X-ray sources in the galaxy, such as X-ray binaries (XRBs) and ultra-luminous $\mathrm{X}$-ray sources (ULXs).

XRBs are off-nuclear X-ray emitting objects that are believed to be powered by the accretion of stars onto compact objects such as stellar-mass black holes and neutron stars. Typically, XRBs have luminosity values in the range of $10^{35-39} \mathrm{erg} \mathrm{s}^{-1}$ (Bogdán \& Gilfanov 2011). They can be classified into high mass XRBs and low mass XRBs, depending on the mass of the companion stars; i.e., $\sim 8 M_{\odot}$ for high mass XRBs and $\sim 2 M_{\odot}$ for low mass XRBs. XRBs can be found in both early and late-type galaxies (Mezcua et al. 2014). However, early-type galaxies are usually dominated by low mass XRBs (Boroson et al. 2011 and Zhang et al. 2012), while late-type star forming galaxies are typically dominated by high mass XRBs (Mezcua et al. 2014).

On the other hand, ULXs are defined as off-nuclear $\mathrm{X}$-ray sources with luminosity values greater than $10^{39} \mathrm{erg}$ $\mathrm{s}^{-1}$, which exceeds the Eddington luminosity for stellar mass black holes (Fabbiano, 2006 and Earnshaw et al. 2019b). Intermediate mass black holes have been long proposed as the candidates for ULXs, however recent studies on several ULXs found that they are actually powered by neutron stars / pulsars (e.g., Bachetti et al. 2014; Fürst et al. 2017; Carpano et al. 2018). ULXs can be found in both elliptical and spiral galaxies, but is more dominant in spirals, most likely due to the higher star formation rates in these galaxies (Swartz et al. 2009; Owen \& Warwick, 2009; Walton et al. 2011).

NGC 1559 has only been observed in X-rays by ROSAT and Chandra. In the ROSAT observation which was conducted in 1995, two ULXs were detected in the spiral arms of the galaxy (Liu \& Bregman, 2005). No other X-ray sources were detected in the observation. In this paper, we will present the Chandra observation of NGC 1559 which has not been published in previous papers, to study the X-ray sources population in the galaxy. The much higher spatial resolution of Chandra as oppose to ROSAT can provide us with a much more detail insight on the X-ray sources population in this galaxy.

We present the details of the Chandra observation, our data reduction, source detection and X-ray spectral analysis procedures in the Methodology Section. In the next section, we present and discuss our results. This is followed by a 
Conclusion Section, where we summarise and conclude our results.

\section{METHODOLOGY}

\section{X-RAY SOURCES IDENTIFICATION}

The observation on NGC 1559 was conducted using the Chandra's Advanced CCD Imaging Spectrometer (ACIS-S) detector with an exposure time of $46.0 \mathrm{ksec}$ (ObsID 16745) on 9 June 2016. The Chandra data was processed using the Chandra Interactive Analysis of Observation (CIAO) to create cleaned event files (level 2) and a new bad pixel file with updated calibration modification using the CHANDRA_REPRO task. From the level 2 event files, an image file is created at energy range 0.5 to $8.0 \mathrm{keV}$ using DMCOPY and FLUXIMAGE tasks, and exposuremap created at energy of $2.5 \mathrm{keV}$. The exposure-map was created using the MKPSFMAP task, with the Enclose Count Fraction (ECF) parameter set to 0.9. Finally, the X-ray sources were detected using the WAVDETECT task at 1 , 2, 4 and 16 pixels. We then overlapped the X-ray sources detected by WAVDETECT on the European Southern Observatory (ESO) Digitized Sky Survey (DSS) optical image of the galaxy to determine sources that are located within the optical boundary of the galaxy.

\section{DETERMINATION OF SOURCES LUMINOSITIES}

The detected sources can be classified into XRB and ULX candidates based upon their calculated luminosities. The source fluxes were first determined based on their detected count rates using the Portable Interactive Multi-Mission Simulator (WEBPIMMS) assuming a simple power-law model, with a photon index of 1.9 , absorbed by Galactic absorption, $\mathrm{N}_{\mathrm{H}}=2.16 \times 10^{20} \mathrm{~cm}^{-2}$ (Kalberla et al. 2005). The source luminosities were then calculated based on the measured fluxes, assuming that they are located within NGC 1559. We classify those with $L<10^{39} \mathrm{erg} \mathrm{s}^{-1}$ as XRB candidates, and those with $L \geq 10^{39} \mathrm{erg} \mathrm{s}^{-1}$ as ULX candidates.

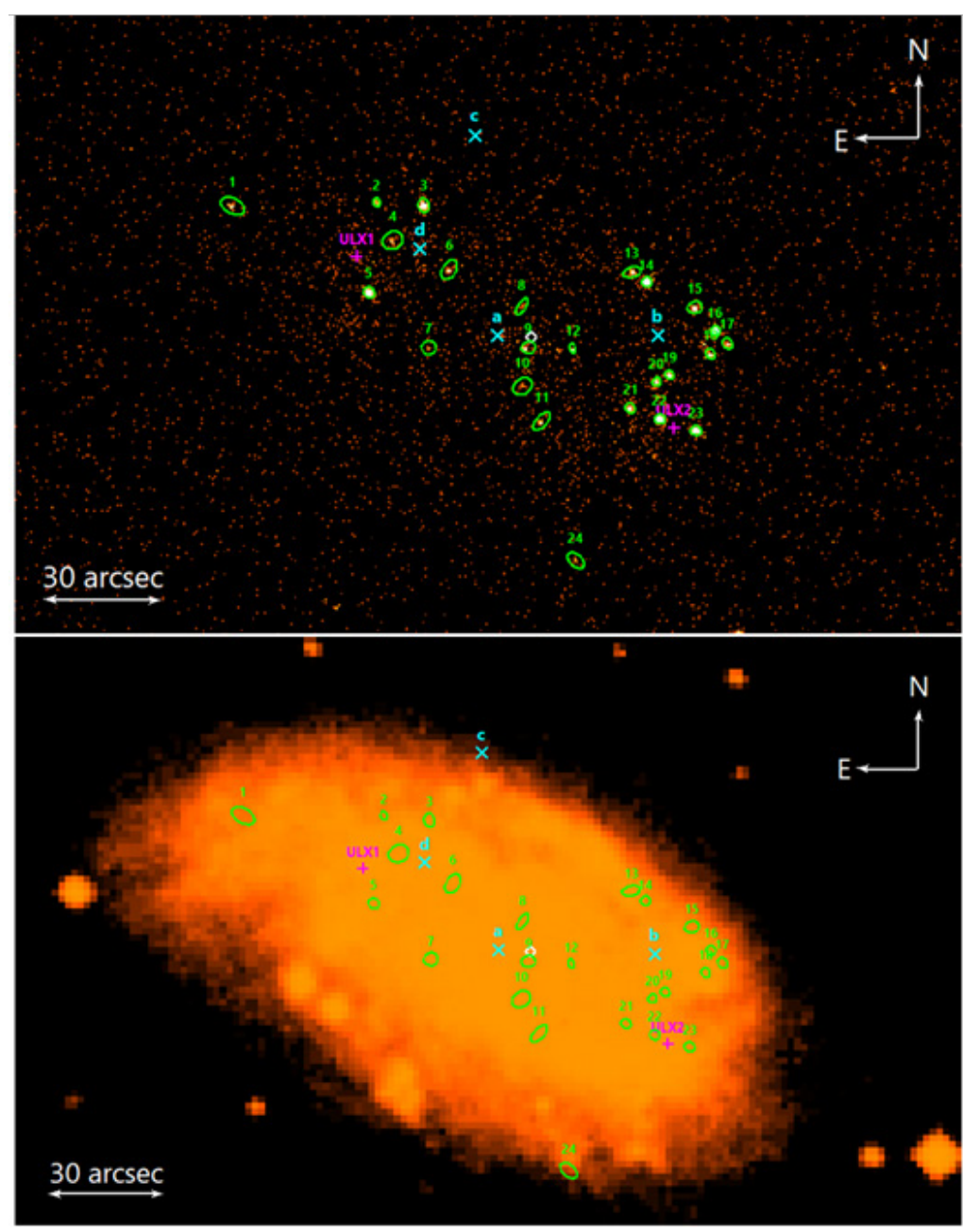

FIGURE 1. X-ray (0.5-8 keV) and optical images of NGC 1559 taken by Chandra (top) and ESO DSS (bottom), respectively.

The detected X-ray sources are marked with green circles. White diamond indicates the center of the galaxy, purple plus markers show the ULXs detected by Liu and Bregman (2005) in the ROSAT observation, and cyan crosses indicate the location of the supernovae detected in the galaxy (a, b, c, and d are SNe 1984J, SNe 1986L, SN 2005df and SN 2009ib, respectively). 
In order to further study the properties of the X-ray sources detected, we performed X-ray spectral analysis for those with relatively high number of counts $(\geq 200$ photon counts) so that we can make reliable spectral analyses. We created the source and background regions for each source, respectively. The spectra were then extracted using the SPECEXTRACT task in CIAO, and grouped to 20 counts per energy bin using GRPPHA task. We then fitted the spectra using the X-ray spectral fitting package, XSPEC v12.10.1. The spectral fittings were optimised using the chi-squared $\left(\chi^{2}\right)$ statistic. The spectra were fitted using two commonly used models to simulate ULXs spectra: (1) a cut-off power-law model, absorbed by the Galactic absorption and line-of-sight obscuration intrinsic to the source (PHABS*TBABS*CUTOFF); and (2) a multicolor blackbody accretion disk model, also absorbed by the Galactic and intrinsic absorption of the source (PHABS*TBABS*DISKPBB). The cut-off power-law model can indicate the energy at which the source spectra turn down at high energy, $E_{\text {cut }}$ while the DISKPBB model can inferred the temperature disk profile, $p$ and inner disk temperature, $T_{\text {in }}$ of the sources. For both models, we fixed the value of Galactic column density to $N_{\mathrm{H}}=2.16 \times 10^{20}$ $\mathrm{cm}^{-2}$ (Kalberla et al. 2005). We calculated the absorbed $0.5-$ $8 \mathrm{keV}$ luminosities of the sources using the fluxes obtained from the best fitting models.
In order to investigate the presence of AGN in NGC 1559, we complement our X-ray analysis on the galaxy with midinfrared data taken from WISE. AGN could be unidentified in X-ray and optical wavelengths due to obscuration and/ or contamination by the dust surrounding the AGN (i.e., the torus) or host galaxy absorption. The majority of the infrared continuum emission from an AGN comes from the torus due to reprocessed emission from the central region. This wavelength has a relatively high optical depth, meaning that it does not significantly suffer from absorption. Therefore, it can be used to detect AGN that are suffering from significant obscuration along our line-of-sight. We used the three-band WISE colour diagram ( $W 1, W 2$ and $W 3$ bands; i.e., 3.4, 4.6 and $12 \mu \mathrm{m}$, respectively) built by Mateos et al. (2012) to determine the presence of AGN at this wavelength (Figure 4).

\section{RESULTS AND DISCUSSION}

X-RAY SOURCES POPULATION

Based on our analysis, twenty-four X-ray sources were found to be located within NGC 1559. Figure 1 shows the optical and Chandra image of the galaxy, showing the detected $\mathrm{X}$-ray sources. Table 1 list the sources, their positions, count rates, fluxes and luminosities.

TABLE 1. X-ray sources detected in NGC 1559 by Chandra.

\begin{tabular}{|c|c|c|c|c|c|c|c|}
\hline $\begin{array}{l}\text { (1) } \\
\text { X-ray source } \\
\text { ID }\end{array}$ & $\begin{array}{c}(2) \\
\mathrm{RA} \\
\mathrm{h} \mathrm{m} \mathrm{s} \\
\end{array}$ & $\begin{array}{c}\text { (3) } \\
\text { Dec } \\
\mathrm{dm} \mathrm{s} \\
\end{array}$ & $\begin{array}{c}\text { (4) } \\
C R_{0.5-8 \mathrm{keV}} \\
\times 10^{-3} \text { counts }^{-1} \\
\end{array}$ & $\begin{array}{c}(5) \\
f_{0.5-8 \mathrm{keV}} \\
\times 10^{-14} \mathrm{erg} \mathrm{cm}^{-2} \mathrm{~s}^{-1}\end{array}$ & $\begin{array}{c}\text { (6) } \\
\log \mathrm{L}^{\mathrm{c}} 0.5-8 \mathrm{keV} \\
\mathrm{erg} \mathrm{s}^{-1} \\
\end{array}$ & $\begin{array}{c}(7) \\
\log \mathrm{L}^{\mathrm{s}} 0.5-8 \mathrm{keV} \\
\mathrm{erg} \mathrm{s}^{-1} \\
\end{array}$ & $\begin{array}{c}\text { (8) } \\
\text { Classification } \\
\text { (candidates) }\end{array}$ \\
\hline 1 & 041746.900 & -624627.664 & $1.32 \pm 0.18$ & $2.59 \pm 0.22$ & $38.50_{-0.05}^{+0.06}$ & $\ldots$ & $\mathrm{XRB}$ \\
\hline 2 & 041741.498 & -624626.727 & $0.17 \pm 0.09$ & $0.34 \pm 0.11$ & $37.92_{-0.12}^{+0.10}$ & $\ldots$ & $\mathrm{XRB}$ \\
\hline 3 & 041739.745 & -624627.537 & $9.03 \pm 0.46$ & $11.5 \pm 0.57$ & $39.35 \pm 0.02$ & $>38.98$ & ULX \\
\hline 4 & 041740.899 & -624636.527 & $0.66 \pm 0.11$ & $1.30 \pm 0.14$ & $38.20_{-0.08}^{+0.07}$ & $\ldots$ & $\mathrm{XRB}$ \\
\hline 5 & 041738.748 & -624649.864 & $11.044_{-0.51}^{+}$ & $14.8 \pm 0.62$ & $39.46 \pm 0.02$ & $>39.28$ & ULX \\
\hline 6 & 041738.788 & -624644.042 & $0.92 \pm 0.15$ & $1.81 \pm 0.19$ & $38.35_{-0.08}^{+0.07}$ & $\ldots$ & $\mathrm{XRB}$ \\
\hline 7 & 041739.557 & -624704.037 & $0.35 \pm 0.09$ & $0.69 \pm 0.11$ & $37.86_{-0.15}^{+0.11}$ & $\ldots$ & $\mathrm{XRB}$ \\
\hline 8 & 041736.068 & -624653.493 & $0.32 \pm 0.07$ & $0.43 \pm 0.08$ & $37.80_{-0.12}^{+0.10}$ & $\ldots$ & $\mathrm{XRB}$ \\
\hline 9 & 041735.920 & -624703.953 & $1.16 \pm 0.15$ & $2.25 \pm 0.19$ & $38.43_{-0.06}^{+0.05}$ & $\ldots$ & $\mathrm{XRB}$ \\
\hline 10 & 041736.046 & -624713.974 & $0.35 \pm 0.09$ & $0.68 \pm 0.11$ & $37.95_{-0.12}^{+0.09}$ & $\ldots$ & $\mathrm{XRB}$ \\
\hline 11 & 041735.328 & -624622.874 & $0.79 \pm 0.13$ & $1.53 \pm 0.16$ & $38.25_{-0.08}^{+0.07}$ & $\ldots$ & $\mathrm{XRB}$ \\
\hline 12 & 041734.162 & -624704.241 & $0.19 \pm 0.07$ & $0.38 \pm 0.08$ & $37.76_{-0.14}^{+0.10}$ & $\ldots$ & $\mathrm{XRB}$ \\
\hline 13 & 041731.938 & -624644.737 & $1.36 \pm 0.18$ & $2.63 \pm 0.22$ & $38.52_{-0.06}^{+0.05}$ & $\ldots$ & $\mathrm{XRB}$ \\
\hline 14 & 041731.346 & -624647.185 & $11.0_{-0.48}^{+}$ & $13.5_{-0.59}^{+0.60}$ & $39.41_{-0.02}^{+}$ & $>38.96$ & ULX \\
\hline 15 & 041729.546 & -624653.721 & $1.58 \pm 0.18$ & $3.06 \pm 0.21$ & $38.58 \pm 0.05$ & $\ldots$ & $\mathrm{XRB}$ \\
\hline 16 & 041728.785 & -624659.795 & $10.34 \pm 0.51$ & $14.0 \pm 0.62$ & $39.43 \pm 0.02$ & $>39.29$ & ULX \\
\hline 17 & 041728.326 & -624702.980 & $0.484 \pm 0.11$ & $0.93 \pm 0.14$ & $38.12_{-0.10}^{+0.08}$ & $\ldots$ & $\mathrm{XRB}$ \\
\hline 18 & 041728.966 & -624705.697 & $1.01 \pm 0.15$ & $1.95 \pm 0.19$ & $38.46_{-0.06}^{+0.05}$ & $\ldots$ & $\mathrm{XRB}$ \\
\hline 19 & 041730.495 & -624711.088 & $2.12 \pm 0.22$ & $4.07 \pm 0.27$ & $38.76 \pm 0.04$ & $\ldots$ & $\mathrm{XRB}$ \\
\hline 20 & 041730.976 & -624712.829 & $1.96 \pm 0.22$ & $3.78 \pm 0.27$ & $38.74_{-}^{+} 0.04$ & $\ldots$ & $X R B$ \\
\hline 21 & 041731.962 & -624719.646 & $1.23 \pm 0.18$ & $2.38 \pm 0.22$ & $38.50_{-0.06}^{+0.05}$ & $\ldots$ & $\mathrm{XRB}$ \\
\hline 22 & 041730.853 & -624722.381 & $1.30 \pm 0.57$ & $17.8+0.71$ & $39.54_{-0.02}^{+0}$ & $>39.11$ & ULX \\
\hline 23 & 041729.506 & -624725.307 & $7.10 \pm 0.40$ & $0.93+0.49$ & $39.25 \pm 0.02$ & $>38.95$ & ULX \\
\hline 24 & 041734.007 & -624758.689 & $0.37 \pm 0.09$ & $0.72 \pm^{+} 0.11$ & $37.92_{-0.12}^{+0.10}$ & $\ldots$ & $X R B$ \\
\hline
\end{tabular}

Notes. Column (1) Identification number (ID) of the detected X-ray sources; (2)- (3) right ascension (RA) and declination (Dec) of the sources; (4) 0.5-8 keV detected count rates; (5-6) absorbed 0.5-8 keV fluxes and luminosities determined from the count rates using WEBPIMMS; (7) absorbed 0.5-8 keV luminosities measured from X-ray spectral analysis; and (8) classification of the sources on the basis of their measured luminosities. 

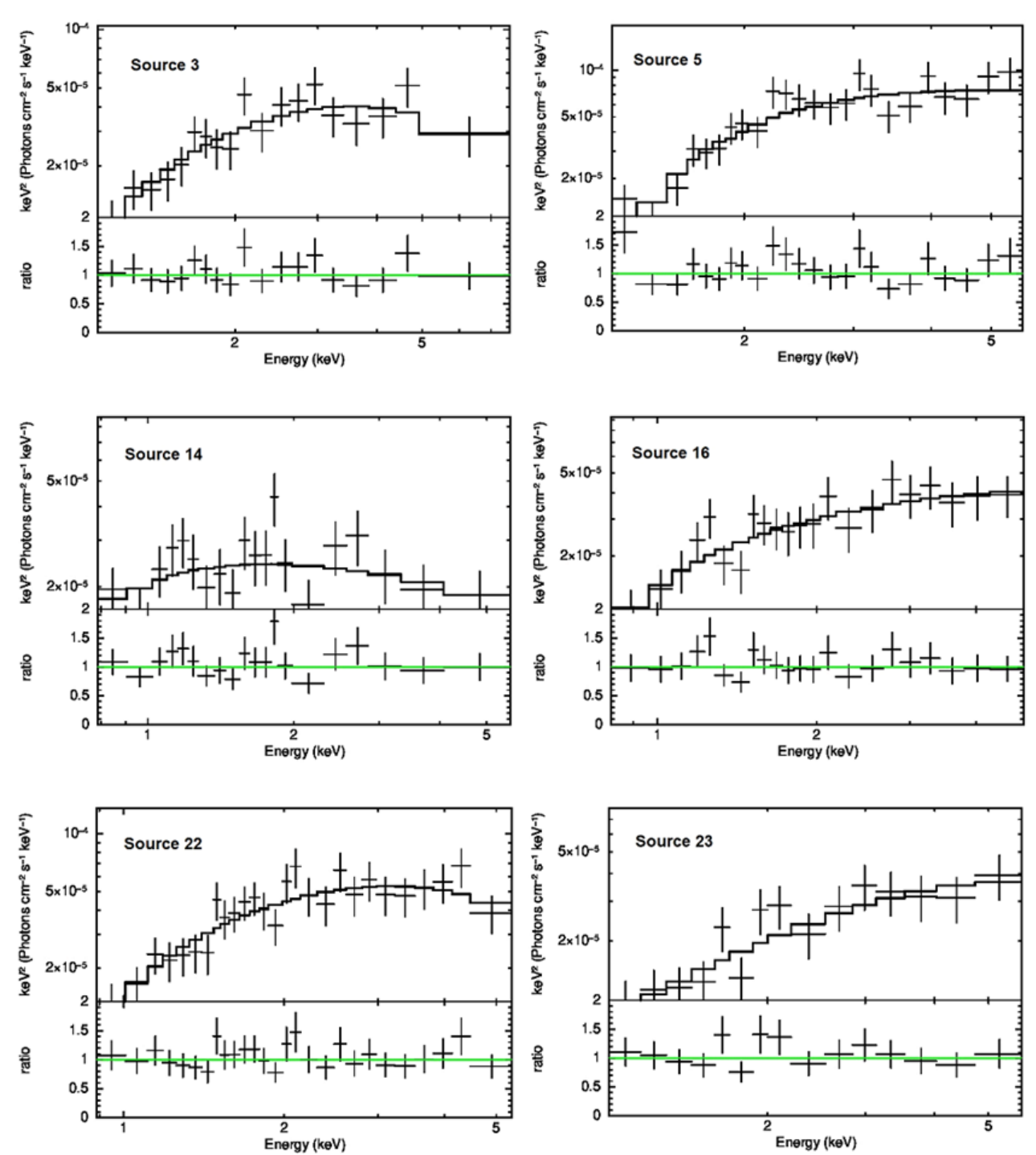

FIGURE 2. The best-fit spectra of the ULX candidates using CUTOFF model. The top panel for each plot shows the data and unfolded model, while the bottom panel shows the ratio between the data and the folded model.

Based on their calculated luminosities, we found that six out of twenty-four sources are potentially ULXs (sources 3, 5, 14, 16, 22 and 23), and the remaining eighteen sources are XRB candidates. Due to the much larger spatial resolution of ROSAT as compared to Chandra, we could not directly associate the two ULXs that were detected in the earlier ROSAT observation (Liu \& Bregman, 2005) with those detected in the Chandra observation. The ULXs may be the same as the two of the six ULXs that we detected using Chandra, or they could actually be the combination of multiple XRBs contributing to a relatively high luminosity that leads them to be classified as ULXs in the ROSAT observation. On the other hand, the two ULXs might have actually decreased in luminosity, making them undetected in the Chandra observation.

\section{SPECTRAL ANALYSIS}

We conducted spectral analysis for sources with count rates of $\geq 200$ to study more about their properties. Unfortunately, there are only six sources with photon counts above this threshold value, and coincidently, all of the six sources are the ULX candidates. Table 2 presents the result of our spectral analysis.

The best fit spectra of each sources using both models are shown in Figure 2 (CUTOFF) and 3 (DISKPBB). In general, our results are not well-constrained due to the relatively low number of counts in the sources spectra. Both the CUTOFF and DISKPBB models gave equally acceptable fit to the spectra of the sources (i.e., reduced chisquared, $\left.\chi_{\mathrm{r}}^{2} \sim 1\right)$.

For the CUTOFF model, we found that the measured photon index values for source 5 and 14 are consistent with the typical value obtained for ULXs; i.e., $\Gamma=2$ (e.g., Annuar et al. 2015 and Earnshaw et al. 2019a). The measured values for source 3, 16, 22 and 23 are $<2$, indicating hard spectra or relatively significant absorption. However, the values are consistent with 2 within their large statistical uncertainties, and the column density values measured for the sources are all $\leq 10^{22} \mathrm{~cm}^{-2}$, suggesting lack of obscuration. This is also 

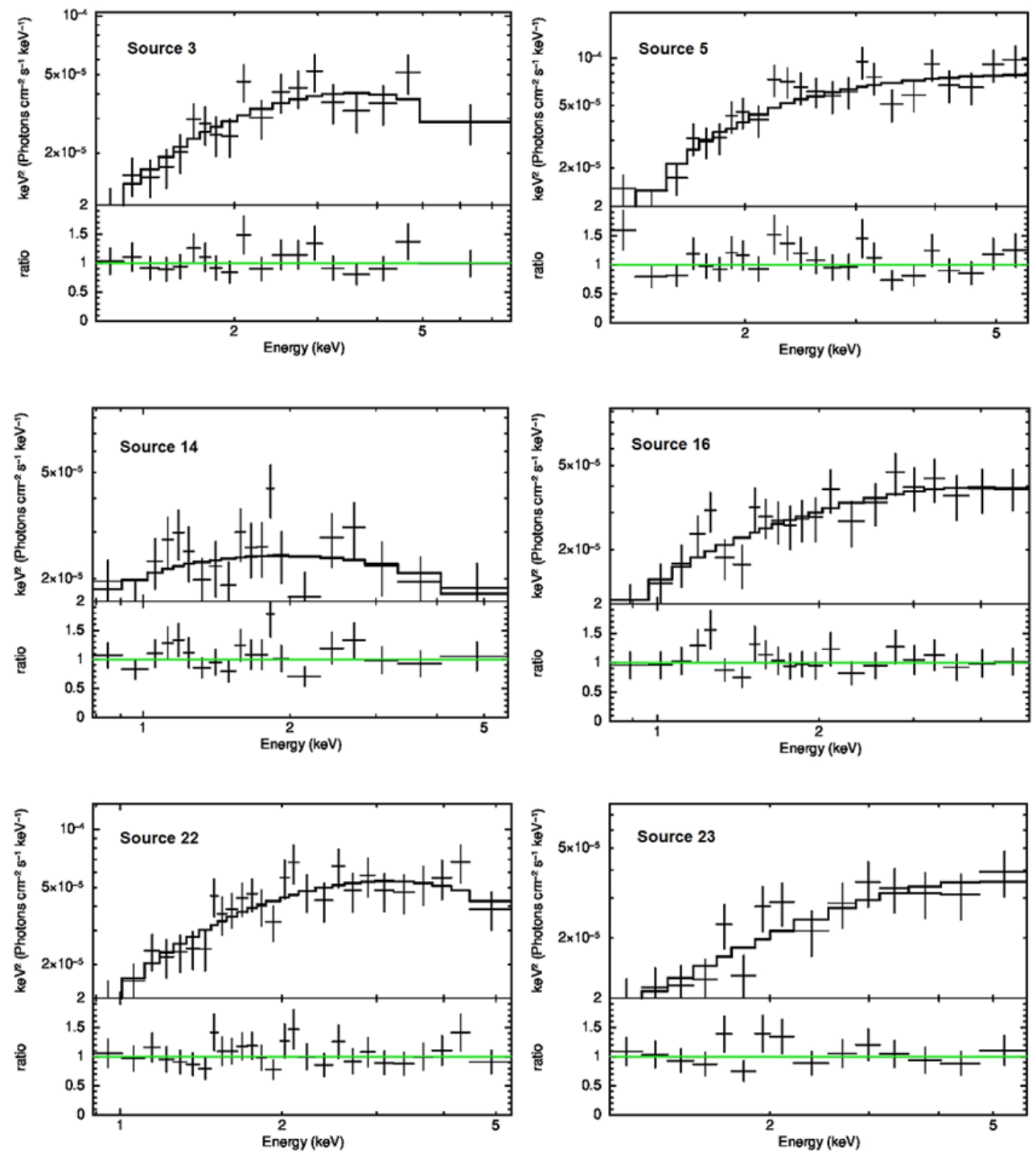

FIGURE 3. The best-fit spectra of the ULX candidates using DISKPBB model. The top panel for each plot shows the data and unfolded model, while the bottom panel shows the ratio between the data and the folded model.

the case for source 14 . Only source 5 has a constraint $N_{\mathrm{H}}$ value of $1.8_{-0.5}^{+0.7} \times 10^{22} \mathrm{~cm}^{-2}$, indicating relatively significant intrinsic absorption towards the source. We could not constraint the values for the high energy cut-off, $E_{\text {cut }}$ for all six sources. However, based on Figure 2, we can see that for source 3, 14 and 22, the spectra seem to drop-off at energy $\sim 5 \mathrm{keV}$. This is consistent with that found in other ULXs in past studies in which they found high energy cut-off at around $10 \mathrm{keV}$ (e.g., Walton et al. 2014; Annuar et al. 2015; Rana et al. 2015).

As for the DISKPBB model, we also could not get a constraint values on the $N_{\mathrm{H}}$ parameter for source 3,14 , 22 and 23. However, the upper limit values are consistent with that obtained from the CUTOFF model. For source 5 and 16 we managed to obtained $N_{\mathrm{H}}$ values of $1.6_{-0.5}^{+0.2} \mathrm{x}$ $10^{22} \mathrm{~cm}^{-2}$ and $2.0 \pm 0.20 \times 10^{22} \mathrm{~cm}^{-2}$, respectively. The $N_{\mathrm{H}}$ value measured for both sources are consistent with that inferred from the CUTOFF model. Meanwhile, only source 22 and 23 have constraint values for the inner disk radius temperature parameter; i.e., $T_{\text {in }}=1.3_{-0.3}^{+4.5} \mathrm{keV}$ and $2.0_{-0.6}^{+1.6}$ $\mathrm{keV}$, respectively. The other sources have lower limit values of $T_{\text {in }} \geq 1$. These values are consistent with the values found for other ULXs in previous studies (e.g., Annuar et al. 2015 and Earnshaw et al. 2019a). We obtained upper limit values for the disk temperature profile for all sources; i.e., $p<0.9$, except for source 23 which we managed to get a measured value of $p=0.8_{-0.3}^{+0.2}$. For source $3,14,16$ and 22 , the upper limit values are $<0.75$, consistent with accretion process dominated by advection (Shakura \& Sunyaev, 1973), suggesting that the nature of these four ULX candidates are stellar-mass black holes undergoing super-Eddington accretion.

The majority of the fluxes we measured for the sources from the spectral fittings are not well-constrained and lower limits. Nevertheless, the luminosities measured from both models are in general not significantly different from each other. We compared the luminosities that we measured from the best-fit model (i.e., $\chi_{r}^{2}$ closest to 1 ) with that estimated from the detected numbers of counts using WEPIMMS in Table 1 . We found that the spectroscopically determined luminosities are consistent with that estimated from the count rates. 
TABLE 2. X-ray spectral fitting results for the ULX candidates

\begin{tabular}{|c|c|c|c|c|c|c|c|c|}
\hline Model & Parameter & Unit & Source 3 & Source 5 & Source 14 & Source 16 & Source 22 & Source 23 \\
\hline \multirow[t]{5}{*}{ CUTOFF } & $\mathrm{N}_{\mathrm{H}} \mathrm{a}$ & $\mathrm{x} 10^{22} \mathrm{~cm}^{-2}$ & $<1.0$ & $1.8_{-0.5}^{+0.7}$ & $<0.3$ & $<0.4$ & $<0.7$ & $<0.6$ \\
\hline & $\Gamma$ & $\ldots$ & $2.2_{-2.5}^{+0.3}$ & $2.2_{-2.5}^{+0.3}$ & $2.0_{-1.1}^{+0.6}$ & $1.2_{-1.5}^{+0.8}$ & $0.2_{-1.2}^{+2.0}$ & $0.3_{-0.7}^{+2.1}$ \\
\hline & $E_{\text {cut }}$ & $\mathrm{keV}$ & $>1.12$ & $>0.01$ & $>1.47$ & $>0.47$ & $>0.8$ & $>1.32$ \\
\hline & $\log L_{0.5-8 \mathrm{keV}}^{\mathrm{b}}$ & $\operatorname{erg~s}^{-1}$ & $39.29_{-0.30}^{+0.01}$ & $>39.28$ & $>38.93$ & $>39.29$ & $39.42_{-0.31}^{+0.03}$ & $>38.95$ \\
\hline & $\chi_{\mathrm{r}}^{2}$ & $\ldots$ & 0.706 & 1.063 & 1.006 & 0.638 & 0.657 & 0.824 \\
\hline \multirow[t]{5}{*}{ DISKPBB } & $\mathrm{N}_{\mathrm{H}}{ }^{\mathrm{a}}$ & $\mathrm{x} 10^{22} \mathrm{~cm}^{-2}$ & $<0.9$ & $1.6_{-0.5}^{+0.2}$ & $<0.2$ & $0.2_{-}^{+} 0.2$ & $<0.7$ & $<0.6$ \\
\hline & $T_{\text {in }}$ & $\mathrm{keV}$ & $>1.1$ & $>1.4$ & $>1.0$ & $>3.0$ & $1.3_{-0.3}^{+4.5}$ & $2.0_{-0.6}^{+1.6}$ \\
\hline & $p$ & $\ldots$ & 0.7 & $<0.9$ & $<0.6$ & 0.5 & 0.7 & $0.8_{-0.3}^{+0.2}$ \\
\hline & $\log L_{0.5-8 \mathrm{keV}}^{\mathrm{b}}$ & $\operatorname{erg~s}^{-1}$ & $>38.98$ & $>39.31$ & $>38.96$ & $>39.07$ & $>39.11$ & $>38.94$ \\
\hline & $\chi_{\mathrm{r}}^{2}$ & $\ldots$ & 0.709 & 1.057 & 1.009 & 0.632 & 0.657 & 0.823 \\
\hline
\end{tabular}

Notes. a Column density intrinsic to the source; $\mathrm{b}$ absorbed $0.5-8 \mathrm{keV}$ luminosity measured from the spectral fitting.

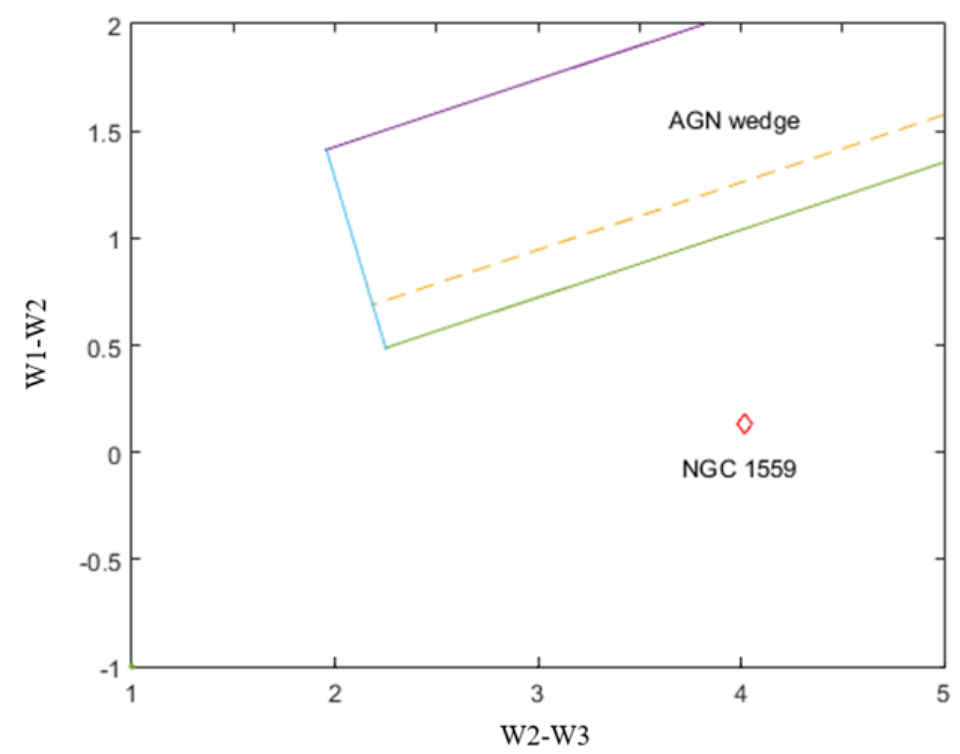

FIGURE 4. Three-band WISE colour diagram (W1, W2 and W3 bands; i.e., 3.4, 4.6 and $12 \mu \mathrm{m}$, respectively) built by Mateos et al.

(2012) to determine the presence of AGN at infrared wavelength. If AGN if presence in a galaxy, it will be located inside the AGN wedge. NGC 1559 (red diamond) is located outside of this wedge, suggesting that it does not host an AGN. This is consistent with the non-detection in X-ray wavelength at the nuclear region of the galaxy, and its HII optical classification.

The total number of X-ray sources found in this galaxy is relatively high for a star formation rate (SFR) of $2.8 M_{\odot}$ $\mathrm{yr}^{-1}$ calculated for this galaxy using its infrared luminosity; i.e., $L_{\mathrm{IR}}=16.2 \times 10^{9} L_{\odot}$ (Goulding \& Alexander, 2009) and relationship derived by Kennicutt (1998). For example, the number of ULXs typically found for galaxies with similar values of SFR is two (e.g, Mapelli et al. 2010). The high number of X-ray sources found however, can be attributed to the relatively high number of supernova occurrence (four) in NGC 1559. Supernova can trigger star formation and form compact stellar remnants, and therefore plays important role in shaping the population of X-ray sources in their host galaxies (e.g., Artale et al. 2019). Based on Figure 1 , we can see that about thirteen of the XRB candidates and all of the ULX candidates are located in or very close to the spiral arms of the galaxy where star formation rate is likely to be relatively high.

\section{AGN DETECTION}

Among the twenty-four X-ray sources detected, none are located at the center of NGC 1559, suggesting the absence of an AGN activity. This is consistent with the ROSAT observation conducted over two decades ago in which they also did not detect any nuclear source in the galaxy. Based on the infrared AGN diagnostic diagram by Mateos et al. (2012) shown in Figure 4, NGC 1559 is located outside of the AGN wedge. This indicates that it does not host an AGN, supporting the HII optical classification of the galaxy and our X-ray analysis result. 


\section{CONCLUSION}

In this paper, we conducted a study on the X-ray sources population in the nearby galaxy NGC 1559 using Chandra telescope. Based on our analysis, we detected twenty-four $\mathrm{X}$-ray sources in the galaxy, of which we classified six as ULX candidates, and eighteen as XRB candidates on the basis of their measured luminosities. We performed X-ray spectral analysis for the six ULX candidates and found that in general, their properties are broadly consistent with that found for other ULXs. The number of X-ray sources found in this galaxy is relatively high as compared to other galaxies with similar star formation rates. This however could be attributed to the relatively high number of supernova explosions in the galaxy. We did not detect any X-ray nuclear point source in the galaxy, suggesting that NGC 1559 does not host an AGN. This is supported by its optical spectral classification and our infrared analysis using WISE.

\section{DECLARATION OF COMPETING INTEREST}

None.

\section{ACKNOWLEDGMENT}

The authors would like to thank the Universiti Kebangsaan Malaysia's grant GGPM-2018-001 for their financial support. The scientific results reported in this article are based on observations made by the Chandra X-ray Observatory and data obtained from the Chandra Data Archive. This research has made use of software provided by the Chandra X-ray Center (CXC) in the application packages CIAO. This research made use of the data obtained through the High Energy Astrophysics Science Archive Research Center (HEASARC) Online Service, provided by the NASA/Goddard Space Flight Center, and the NASA/IPAC extragalactic Database (NED) operated by JPL, Caltech under contract with NASA.

\section{REFERENCES}

Artale M. C., Giacobbo N., Mapelli M. and Esposito P. 2019. The high mass x-ray binaries in star-forming galaxies. International, Astronomical Unioun Symposium 346: 332-336.

Annuar A., Gandhi P., Alexander D. M., Lansbury G. B, Arèvalo P., Ballantyne D. R., Baloković M., et al. 2015. NuSTAR observatios of the compton-thick active galactic nucleus and ultraluminous x-ray source candidate in NGC 5643. The Astrophysical Journal 815: 36.

Bachetti M., Harrison F. A., Walton D. J., Grefenstette B. W., Chakrabarty D., Fürst F., et al. 2014. An Ultraluminous x-ray sources powered by an accreting neutron star. Nature 514:202204.
Beck R., Shoutenkov V., Ehle M., Harnett J. I., Haynes R. F., Shukurov A., Sokoloff D. D. and Thierbach M. 2002. Magnetic field in bared galaxies. Astonomy and Astrophsics 391: 83-102.

Boroson B., Dong-Woo K and Fabbiano G. 2011. Revisiting with chandra the scaling relations of the x-ray emission components (binaries, nuclei and hot gas of early type galaxies. The Astrophysical Journal 792: 12.

Bógdan A. and Gilfanov, M. 2011. Unresolved and diffuse component of $\mathrm{x}$-ray emission and $\mathrm{x} / \mathrm{k}$ luminosity ratio in nearby early-type and late-type galxies. Monthly Notices of the Royal Astronomical Society 418: 1901-1912.

Carpano S., Haberl F., Maitra C., and Vasilopoulos G. 2018. Discovery of pulsations from NGC 300 ULX1 and its fast period evolution. Monthly Notices of the Royal Astronomical Society 476:45-49.

Earnshaw H. P., Brian W. G., Brightman M., Walton D. J., Barret D., Fürst F., et al. 2019a. A broadband Look at the Old and New ULXs of NGC 6946. The Astrophysical Journal 881: 38-57.

Earnshaw H. P., Roberts T.P., Middleton M.J., Walton D. J. n and Mateos S. 2019b. A new, clean catalogue of extragalactic nonnuclear x-ray sources in nearby galaxies. Monthly Notices of the Royal Astronomical Society 483:4.

Evans R., Overbeek D. and Thompson G. 1984. Probable supernova in NGC 1559. International, Astronomical Unioun Circular 3963: 1.

Fabbiano G. 2006. Population of x-ray sources in galaxies. The Annual Review of Astrophysics 44:66-323.

Fürst F., Walton D. J., Stern D., Bachetti M., Barret D., Brightman M., et al. 2017. Astrophysical Journal 834:77.

Goulding A. D. and Alexander D. M. 2009. Towards a complete census of AGN in nearby galaxies: A large population of optically unidentified AGN. Monthly Notices of the Royal Astronomical Society 398:1165-1193.

Kalberla P. M. W., Burton W. B., Hartmann D., Arnal E. M., Bajaja E., Morras R., et al. 2005. The Leiden Argentine Bonn (LAB) Survey of Galactic HI. Final data release of the combined LDS and IAR surveys with improved stray-radiation corrections. Astronomy and Astrophysics 440:75-782.

Kennicutt R. C. Jr. 1998. The global schmidt law in star-forming galaxies. The Astrophysical Journal 498: 541-552.

Liu Ji-Feng and Bregman J. N. 2004. Ultraluminous x-ray sources in nearby galaxies from ROSAT high resolution imager observation I. Data Analysis. The Astrophysical Journal Supplement Series 157: 59-152.

Mack P., Coulson I. M., Cragg T., Evans R. O. and McNaught R. H. 1986. Supernova 1986L in NGC 1559. International Astronomical Unioun Cirulars 4270: 2.

Mapelli M., Ripamonti E., Zampieri L., Colpi M. and Bressan A. 2010. Ultra-luminous X-ray sources and remnants of massive metal-poor stars. Monthly Notices of the Royal Astronomical Society 408:234-253.

Mateos S., Alonso-Herrero A., Carrera F. J., Blain A., Watson M G., Barcons X., et al. 2012. Using the Bright Ultra-Hard XMM-Newton Survey to define an ir slection of lumininous 
AGN based on WISE colours. Monthly Notices of the Royal Astronomical Society 426:3271-3281.

Mezcua M, Fabbiano G., Gladstone J. C., Farrell S. A. and Soria R. 2014. Revealing the nature of the ULX and X-ray population of the spiral galaxy NGC 4088. The Astrophysical Journal 785: 121.

Owen R.A. and Warwick R.S. 2009. X-ray emission from the extended disks of spiral. Monthly Notices of the Royal Astronomical Society. 394: 1741-1757.

Pignata G., Maza J., Hamuy M., Antezana R., Gonzale L., Lopez P., et al. 2009. Supenova 2009ib in NGC 1559. Central Bureau Electronic Telegrams 1902: 1.

Rana V., Harrison F. A., Bachetti M., Walton D. J., Fürst F., Barret D., et al. 2015. The broadband XMM-Newton and NuSTAR $\mathrm{x}$-ray spectra of two ultraluminous $\mathrm{x}$-ray sources in the galaxy IC 342. The Astrophysical Journal 799: 121.

Shakura N. I. and Sunyaev R. A. 1973. Reprint of 1973A\&A....24..337S. Black holes in binary systems. Observational appearance. Astronomy and Astrophysics 500: 33-51.
Swartz D. A., Soria R., Tennan A. F. T. and Yukita M. 2011. a complete ample of ultraluminous $\mathrm{x}$-ray sources host galaxies. The Astrophysical Journal 741: 49-59.

Swartz D. A., Tennant A. F. and Soria R. 2009. Ultraluminous $\mathrm{x}$-ray sources correlations with star-forming regions. The Astrophysical 703: 159-168.

Walton D. J., Harrison F. A., Grefenstette B. W., Miller J. M., Bachetti M., Barret D., et al. 2014. Broadband x-ray spectra of the ultraluminous X-ray source holmberg IX X-1 observed with NuSTAR, XMM-Newton, and Suzaku. The Astrophysical Journal 793: 21 .

Walton D. J., Roberts T. P., Mateos, S. and Heard, V. 2011. 2 XMM ultraluminous x-ray source candidates in nearby galaxies. Monthly Notices of the Royal Astronomical Society 416: 18441861.

Wang L. and Baade D. 2005. Supernovae 2005de and 2005df. Central Bureau Electronic Telegrams 193: 1.

Zhang Z., Gilfanov M. and Bógdan A. 2012. Dependence of the LMXB popultion on stellar age. Astronomy \& Astrophysics 546: 36 . 